\title{
$p$-VALENT CLOSE-TO-CONVEX FUNCTIONS
}

\author{
BY \\ A. E. LIVINGSTON $\left({ }^{1}\right)$
}

1. Introduction. Let $S(p)$ denote the class of functions, which are regular and $p$-valently star-like in $|z|<1$. A function

$$
f(z)=a_{1} z+a_{2} z^{2}+\cdots \quad(|z|<1)
$$

is a member of $S(p)$, if there exists a positive number $\rho$ such that for $\rho<|z|<1$

$$
\operatorname{Re}\left[\frac{z f^{\prime}(z)}{f(z)}\right]>0
$$

and

$$
\int_{0}^{2 \pi} \operatorname{Re}\left[\frac{z f^{\prime}(z)}{f(z)}\right] d \theta=2 p \pi
$$

The class $S(p)$ has been studied previously by Goodman [4], Robertson [9] and others. Goodman [4] has shown that a function in $S(p)$ is p-valent and has exactly $p$ roots in $|z|<1$.

Goodman [4] also defined the class of $p$-valent convex functions, which we will refer to as $C(p)$. A function

$$
f(z)=a_{1} z+a_{2} z^{2}+\cdots \quad(|z|<1)
$$

is said to be in $C(p)$, if there exists a $\rho$ such that for $\rho<|z|<1$

$$
1+\operatorname{Re}\left[\frac{z f^{\prime \prime}(z)}{f^{\prime}(z)}\right]>0
$$

and

$$
\int_{0}^{2 \pi}\left[1+\operatorname{Re} \frac{z f^{\prime \prime}(z)}{f^{\prime}(z)}\right] d \theta=2 p \pi
$$

A function in $C(p)$ is at most $p$-valent and has $(p-1)$ critical points in $|z|<1 . S(p)$ and $C(p)$ are related to each other in the same way as $S(1)$ and $C(1)$. Namely, $f(z)$ is in $C(p)$ if and only if $z f^{\prime}(z)$ is in $S(p)$.

Kaplan [5] defined the class of close-to-convex functions. A function $F(z)$,

Received by the editors August 23, 1963 and, in revised form, December 5, 1963.

( $\left.{ }^{1}\right)$ This paper is part of a doctoral thesis written under the direction of Professor M. S. Robertson. 
regular for $|z|<1$, with $F(0)=0$ and $F^{\prime}(0) \neq 0$ is said to be close-to-convex if there exists $\phi(z)$ in $C(1)$ such that

$$
\operatorname{Re}\left[\frac{F^{\prime}(z)}{\phi^{\prime}(z)}\right]>0 \quad(|z|<1) .
$$

Notice that we may rewrite the last inequality to read

$$
\operatorname{Re}\left[\frac{z F^{\prime}(z)}{f(z)}\right]>0 \quad(|z|<1)
$$

for some function $f(z)$ in $S(1)$.

Umezawa [13] extended this definition to the case of $p$-valent functions. According to Umezawa, a function

$$
F(z)=z^{q}+a_{q+1} z^{q+1}+\cdots \quad(|z|<1)
$$

is $p$-valently close-to-convex, if there exists

$$
\phi(z)=z^{q}+b_{q+1} z^{q+1}+\cdots \quad(|z|<1)
$$

in $C(p)$ such that

$$
\operatorname{Re}\left[\frac{F^{\prime}(z)}{\phi^{\prime}(z)}\right]>0 \quad(|z|<1) .
$$

It is known that a function in this class is at most $p$-valent in $|z|<1$ [13].

However, Umezawa's definition requires that the zeros of $F^{\prime}(z)$ and $\phi^{\prime}(z)$ have the same positions and multiplicities. We will redefine the concept of a close-to-convex function by requiring that (1.5) should hold only in some range $\rho<|z|<1$. Furthermore, we will not require that our functions be normalized.

Definition. We shall say that a function

$$
F(z)=a_{1} z+a_{2} z^{2}+\cdots \quad(|z|<1),
$$

regular for $|z|<1$, is $p$-valently close-to-convex, or is in $\mathscr{K}(p)$, if it satisfies one of the following 'conditions.

(A) There exists a function $f(z)$ in $S(p)$ and a positive number $\rho$ such that

$$
\operatorname{Re}\left[\frac{z F^{\prime}(z)}{f(z)}\right]>0 \quad(\rho<|z|<1) .
$$

(B) $F(z)$ is regular on $|z|=1$ and there exists a function $f(z)$ in $S(p)$, also regular on $|z|=1$, such that (1.6) holds on $|z|=1$.

Notice that if $F(z)$ satisfies (A), then there exists a $\delta$ such that $G(z)=F(\beta z)$ satisfies (B) for $\delta<\beta<1$.

If $F(z)$ is in $S(p)$, then taking $f(z)=F(z)$, we see that $F(z)$ is in $\mathscr{K}(p)$. Also, if $F(z)$ is in $C(p)$, then taking $f(z)=z F^{\prime}(z)$, we see that $F(z)$ is in $\mathscr{K}(p)$. 
In $\$ 2$ we will show that a function in $\mathscr{K}(p)$ is at most $p$-valent in $|z|<1$. We are also able to obtain sufficient conditions for a function $F(z)$ to be in $\mathscr{K}(p)$, provided $F(z)$ is regular on $|z|=1$ : If $F(z)$ has $p$ zeros at the origin, then we are able to remove the condition of regularity on $|z|=1$.

Considerable interest has been shown in the coefficient problem for functions, which are at most $p$-valent in $|z|<1$. Goodman [3] has conjectured that if

$$
F(z)=a_{1} z+a_{2} z^{2}+\cdots \quad(|z|<1)
$$

is regular and at most $p$-valent in $|z|<1$, then

$$
\left|a_{n}\right|<\sum_{k=1}^{p} \frac{2 k(n+p) !}{(p+k) !(p-k) !(n-p-1) !\left(n^{2}-k^{2}\right)}\left|a_{k}\right|
$$

for $n>p$.

The conjecture was proven by Goodman and Robertson [2] for a function in $S(p)$, in case all its coefficients are real and by Robertson [9] for $F(z)$ in $S(p)$, in case $a_{1}=a_{2}=\cdots=a_{p-2}=0$, the remaining coefficients being complex. In $\$ 3$ we will prove the conjecture for the $(p+1)$ st coefficient of an arbitrary function in $\mathscr{K}(p)$. This is the largest class of $p$ valent functions for which the exact bound on the $(p+1)$ st coefficient is known. We also obtain some sharp upper and lower bounds on $\left|F^{\prime}(z)\right|$ for $F(z)$ in $\mathscr{K}(p)$.

$\$ 4$ deals with the radii of close-to-convexity and convexity for a function in $\mathscr{K}(p)$. If

$$
F(z)=a_{q} z^{q}+a_{q+1} z^{q+1}+\cdots \quad(|z|<1)
$$

is in $\mathscr{K}(p)$, then we obtain a $r_{q}<1$ such that $F(z)$ is $q$-valently close-toconvex in $|z|<r_{q}$ and $\beta_{q}<1$ such that $F(z)$ is $q$-valently convex in $|z|<\beta_{q}$. The numbers $r_{q}$ and $\beta_{q}$ depend upon the nonzero critical points of $F(z)$. We are able to show that the number $\beta_{q}$ gives us the best possible result. However, we are not able to show this for the number $r_{q}$.

2. The class $\mathscr{K}(p)$. We will make use of the following lemma due to Umezawa [12].

Lemma 1. Let $f(z)$ be regular for $|z| \leqq r$ and $f^{\prime}(z) \neq 0$ on $|z|=r$. Suppose that for $z=r e^{i \theta}$

$$
\int_{0}^{2 \pi} d \arg d f(z)=\int_{0}^{2 \pi} \frac{\partial}{\partial \theta}\left[\arg z f^{\prime}(z)\right] d \theta=\int_{0}^{2 \pi} \operatorname{Re}\left[1+\frac{z f^{\prime \prime}(z)}{f^{\prime}(z)}\right] d \theta=2 p \pi\left(^{2}\right) .
$$

If, furthermore,

$\left({ }^{2}\right)$ Geometrically this says that the angle that the tangent to the image of $|z|=r$ makes with the positive $x$-axis goes through a change of $2 p \pi$ as $z$ traverses $|z|=r$. In other words, the image of $|z|=r$, under $w=f(z)$, makes $p$-loops. 


$$
\int_{\theta_{1}}^{\theta_{2}} d \arg d f(z)=\int_{\theta_{1}}^{\theta_{2}} \frac{\partial}{\partial \theta}\left[\arg z f^{\prime}(z)\right] d \theta>-\pi \text { for } \theta_{1}<\theta_{2},
$$

then $f(z)$ is at most p-valent in $|z|<r$.

Theorem 1. If $F(z)$ is in $\mathscr{K}(p)$, then $F(z)$ is at most p-valent in $|z|<1$.

Proof. There exists $f(z)$ in $S(p)$ and $\rho<1$ such that

$$
\operatorname{Re}\left[\frac{z F^{\prime}(z)}{f(z)}\right]>0 \quad(\rho<|z|<1)
$$

Since $z F^{\prime}(z) / f(z) \neq 0$ and $z F^{\prime}(z) \neq 0$ for $|z|=r(\rho<r<1)$, we may define $\arg \left[z F^{\prime}(z) / f(z)\right]$ and $\arg \left[z F^{\prime}(z)\right]$ to be single-valued and continuous on $|z|=r$. Since $f(z)=\left[f(z) / z F^{\prime}(z)\right]\left[z F^{\prime}(z)\right]$, then $\arg f(z)=\arg \left[z F^{\prime}(z)\right]$ $-\arg \left[z F^{\prime}(z) / f(z)\right]$ will be uniquely determined and by (2.1) we have for $z=r e^{i \theta}(\rho<r<1)$,

$$
-\frac{\pi}{2}<\arg z F^{\prime}(z)-\arg f(z)<\frac{\pi}{2}
$$

Let $\theta_{1}<\theta_{2}$, then

$$
-\frac{\pi}{2}<\arg r e^{i \theta_{2}} F^{\prime}\left(r e^{i \theta_{2}}\right)-\arg f\left(r e^{i \theta_{2}}\right)<\frac{\pi}{2}
$$

and

$$
-\frac{\pi}{2}<-\arg r e^{i \theta_{1}} F^{\prime}\left(r e^{i \theta_{1}}\right)+\arg f\left(r e^{i \theta_{1}}\right)<\frac{\pi}{2} .
$$

Combining (2.2) and (2.3), we obtain

$$
\begin{aligned}
-\pi & +\arg f\left(r e^{i \theta_{2}}\right)-\arg f\left(r e^{i \theta_{1}}\right) \\
& <\arg \left[r e^{i \theta_{2}} F^{\prime}\left(r e^{i \theta_{2}}\right)\right]-\arg \left[r e^{i \theta_{1}} F^{\prime}\left(r e^{i \theta_{1}}\right)\right] \\
& <\pi+\arg f\left(r e^{i \theta_{2}}\right)-\arg f\left(r e^{i \theta_{1}}\right)
\end{aligned}
$$

or

$$
\begin{aligned}
-\pi+\int_{\theta_{1}}^{\theta_{2}} d \arg f\left(r e^{i \theta}\right) & <\int_{\theta_{1}}^{\theta_{2}} d \arg d F\left(r e^{i \theta}\right) \\
& <\pi+\int_{\theta_{1}}^{\theta_{2}} d \arg f\left(r e^{i \theta}\right) .
\end{aligned}
$$

Since $f(z)$ is in $S(p)$,

$$
\int_{\theta_{1}}^{\theta_{2}} d \arg f\left(r e^{i \theta}\right)>0 .
$$

Thus the left side of (2.5) gives 


$$
\int_{\theta_{1}}^{\theta_{2}} d \arg d F\left(r e^{i \theta}\right)>-\pi .
$$

Taking $\theta_{1}=0$ and $\theta_{2}=2 \pi$ in (2.5) and using the fact that

$$
\int_{0}^{2 \pi} d \arg f\left(r e^{i \theta}\right)=2 p \pi
$$

we obtain

$$
(2 p-1) \pi<\int_{0}^{2 \pi} d \arg d F\left(r e^{i \theta}\right)<(2 p+1) \pi .
$$

However, the integral in (2.7) is an integral multiple of $2 \pi$. Therefore,

$$
\int_{0}^{2 \pi} d \arg d F\left(r e^{i \theta}\right)=2 p \pi .
$$

Thus, by Lemma $1, F(z)$ is at most $p$-valent for $|z|<r$. Since $r$ was arbitrary $(\rho<r<1), F(z)$ is at most $p$-valent for $|z|<1$.

Since (2.8) holds for any function in $\mathscr{K}(p)$ for some range $\rho<|z|<1$, we easily obtain the following corollary.

CoRollary. If $F(z)$ is in $\mathscr{K}(p)$, then $F^{\prime}(z)$ has exactly $(p-1)$ zeros in $|z|<1$.

Necessary and sufficient conditions for a function $F(z)$, regular in $|z|<1$, with $F(0)=0$ and $F^{\prime}(z) \neq 0$ to be in $\mathscr{K}(1)$ have been given by Kaplan [5]. We see from the proof of Theorem 1 that necessary conditions for $F(z)$ to be in $\mathscr{K}(p)$ are that (2.6) and (2.8) hold in some range $\rho<|z|<1$. We will now show these conditions to be sufficient in two particular cases. The method of proof used is that established by Kaplan [5].

LEMMA 2. Let

$$
F(z)=a_{p} z^{p}+a_{p+1} z^{p+1}+\cdots
$$

be regular for $|z| \leqq 1$. If

$$
\int_{0}^{2 \pi} d \arg d F(z)=2 p \pi
$$

and

$$
\int_{\theta_{1}}^{\theta_{2}} d \arg d F(z)>-\pi \quad\left(\theta_{1}<\theta_{2}\right)
$$

for $|z|=1$, then $F(z)$ is in $\mathscr{K}(p)$.

REMARK. We will show that there exists a function $f(z)$ in $S(p)$ with all its zeros at the origin, which is regular for $|z|<1+\epsilon$ for some $\epsilon>0$, and 
such that $\operatorname{Re}\left[z F^{\prime}(z) / f(z)\right]>0$ for $|z|<1+\epsilon$. This is actually more than we need to prove the lemma, but it is needed in the proof of Theorem 3.

Proof. Since $F(z)$ is regular on $|z|=1$, it is regular in some circle containing $|z| \leqq 1$. By continuity we then have the existence of some $\epsilon>0$ such that (2.9) and (2.10) hold for $1 \leqq|z| \leqq(1+\epsilon)$. Now, the function $z^{(1-p)} F^{\prime}(z)$ is free of zeros in $|z| \leqq(1+\epsilon)$. Hence, we may define $\arg z^{(1-p)} F^{\prime}(z)$ to be single-valued and continuous in $|z| \leqq 1+\epsilon$.

Let

$$
p(r, \theta)=\arg \left[\left(r e^{i \theta}\right)^{(1-p)} F^{\prime}\left(r e^{i \theta}\right)\right] \quad(r \leqq 1+\epsilon)
$$

and

$$
P(r, \theta)=p(r, \theta)+p \theta .
$$

Then, since (2.9) and (2.10) hold for $|z|=1+\epsilon$, we have

$$
\begin{aligned}
P(1+\epsilon, \theta+2 \pi)-P(1+\epsilon, \theta) & =2 p \pi, \\
P\left(1+\epsilon, \theta_{2}\right)-P\left(1+\epsilon, \theta_{1}\right) & >-\pi \text { for } \theta_{1}<\theta_{2} .
\end{aligned}
$$

Using an argument identical to Kaplan's [5], we may show the existence of a function $S(1+\epsilon, \theta)$, which is increasing in $\theta$ and such that

$$
S(1+\epsilon, \theta+2 \pi)-S(1+\epsilon, \theta)=2 p \pi
$$

and

$$
|S(1+\epsilon, \theta)-P(1+\epsilon, \theta)| \leqq \frac{\pi}{2}
$$

Let

$$
q(r, \theta)=\frac{1}{2 \pi} \int_{0}^{2 \pi} \frac{\left[(1+\epsilon)^{2}-r^{2}\right][S(1+\epsilon, \alpha)-p \alpha] d \alpha}{(1+\epsilon)^{2}+r^{2}-2(1+\epsilon) r \cos (\alpha-\theta)} .
$$

Then, $q(r, \theta)$ is harmonic for $r<1+\epsilon$.

Let $Q(r, \theta)=q(r, \theta)+p \theta$ for $r<1+\epsilon$. Using the fact that $S(1+\epsilon, \alpha)$ $-p \alpha$ has period $2 \pi$, we obtain for $r<1+\epsilon$ and $\theta_{1}<\theta_{2}$,

$$
\begin{aligned}
Q\left(r, \theta_{2}\right) & -Q\left(r, \theta_{1}\right) \\
& =\int_{0}^{2 \pi} \frac{\left[(1+\epsilon)^{2}-r^{2}\right]\left[S\left(1+\epsilon, \alpha+\theta_{2}\right)-S\left(1+\epsilon, \alpha+\theta_{1}\right)\right] d \alpha}{(1+\epsilon)^{2}+r^{2}-2(1+\epsilon) r \cos \alpha} .
\end{aligned}
$$

Since $S(1+\epsilon, \alpha)$ is increasing

$$
Q\left(r, \theta_{2}\right)-Q\left(r, \theta_{1}\right) \geqq 0 .
$$

Thus $(\partial / \partial \theta) Q(r, \theta) \geqq 0$ for $r<1+\epsilon$.

Let $h(z)$ be a function, regular for $|z|<1+\epsilon$, such that $\operatorname{Im}\left[h\left(r e^{i \theta}\right)\right]$ $=q(r, \theta)$ and let 


$$
f(z)=z^{p} e^{h(z)}=b_{p} z^{p}+\cdots \quad(|z|<1+\epsilon) .
$$

For $|z|<1+\epsilon$,

$$
\operatorname{Re}\left[\frac{z f^{\prime}(z)}{f(z)}\right]=\frac{\partial}{\partial \theta} \arg f(z)=\frac{\partial}{\partial \theta}(p \theta+q(r, \theta))=\frac{\partial}{\partial \theta} Q(r, \theta) \geqq 0 .
$$

But $z f^{\prime}(z) / f(z)$ is regular for $|z|<1+\epsilon$. Thus,

$$
\operatorname{Re}\left[\frac{z f^{\prime}(z)}{f(z)}\right]>0 \text { for }|z|<1+\epsilon .
$$

Since $f(z)$ has $p$ zeros, all of them at the origin,

$$
\int_{0}^{2 \pi} \operatorname{Re}\left[\frac{z f^{\prime}(z)}{f(z)}\right] d \theta=2 p \pi \quad(|z|<1+\epsilon) .
$$

Hence, $f(z)$ is $p$-valently star-like for $|z|<1+\epsilon$.

Now, for $z=r e^{i \theta}, r<1+\epsilon$, we have

$$
\begin{aligned}
\left|\arg \frac{z F^{\prime}(z)}{f(z)}\right| & =\left|\arg z F^{\prime}(z)-\arg f(z)\right| \\
& =|P(r, \theta)-q(r, \theta)-p \theta| \\
& =|p(r, \theta)-q(r, \theta)| .
\end{aligned}
$$

Since $p(r, \theta)$ is harmonic for $|z|<1+\epsilon$, we may write

$$
p(r, \theta)=\frac{1}{2 \pi} \int_{0}^{2 \pi} \frac{\left[(1+\epsilon)^{2}-r^{2}\right] p(1+\epsilon, \alpha)}{(1+\epsilon)^{2}+r^{2}-2(1+\epsilon) r \cos (\alpha-\theta)} d \alpha .
$$

Then, using (2.12), (2.13) and (2.14), we obtain

$$
\begin{aligned}
\left|\arg \frac{z F^{\prime}(z)}{f(z)}\right| & =|p(r, \theta)-q(r, \theta)| \\
& =\frac{1}{2 \pi}\left|\int_{0}^{2 \pi} \frac{\left[(1+\epsilon)^{2}-r^{2}\right][P(1+\epsilon, \alpha)-S(1+\epsilon, \alpha)] d \alpha}{(1+\epsilon)^{2}+r^{2}-2(1+\epsilon) r \cos (\alpha-\theta)}\right| \\
& \leqq \frac{\pi}{2} .
\end{aligned}
$$

Thus $\operatorname{Re}\left[z F^{\prime}(z) / f(z)\right] \geqq 0$ for $|z|<1+\epsilon$. Hence, either $\operatorname{Re}\left[z F^{\prime}(z) / f(z)\right]>0$ for $|z|<1+\epsilon$, in which case $F(z)$ is in $\mathscr{K}(p)$, or $z F^{\prime}(z) / f(z)$ reduces to a constant for $|z|<1+\epsilon$. In the second case $F(z)$ is in $C(p) \subset \mathscr{K}(p)$.

TheOREM 2. Let

$$
F(z)=a_{p} z^{p}+a_{p+1} z^{p+1}, \cdots \quad(|z|<1)
$$

be regular for $|z|<1$. If (2.9) and (2.10) hold for some range $\rho<|z|<1$, then $F(z)$ is in $\mathscr{K}(p)$.

Proof. Let $\rho<\delta<1$. Then the function $G_{\delta}(z)=F(\delta z)$ is regular on $|z|=1$ 
and satisfies (2.9) and (2.10) on $|z|=1$. Hence, by Lemma 2, $G_{\delta}(z)$ is in $\mathscr{K}(p)$ and there exists

$$
f_{\delta}(z)=b_{p} z^{p}+\cdots \quad(|z|<1)
$$

in $S(p)$ such that

$$
\operatorname{Re}\left[\frac{z G_{\delta}^{\prime}(z)}{f_{\delta}(z)}\right]>0 \quad(|z|<1) .
$$

We may assume that $\left|b_{p}\right|=1$. Cartwright [1] has shown that the family of $p$-valent functions with the moduli of the first $p$ coefficients fixed is a normal family. Thus we may choose a sequence $\delta_{n}$ tending to 1 , such that the sequence of functions $f_{\delta_{n}}(z)$ tends to a function $f(z)$ in $S(p)$. Since $z G_{\delta_{n}}^{\prime}(z)$ tends to $z F^{\prime}(z)$, we obtain from (2.15) that

$$
\operatorname{Re}\left[\frac{z F^{\prime}(z)}{f(z)}\right] \geqq 0 \text { for }|z|<1
$$

This implies that $F(z)$ is in $\mathscr{K}(p)$.

THEOREM 3. Let

$$
F(z)=a_{q} z^{q}+\cdots \quad(1 \leqq q \leqq p)
$$

be regular for $|z| \leqq 1$. If (2.9) and (2.10) hold on $|z|=1$, then $F(z)$ is in $\mathscr{K}(p)$.

Proof. By condition (2.9) $F^{\prime}(z)$ has $(p-1)$ zeros in $|z|<1,(q-1)$ of them at the origin. Let $\alpha_{1}, \alpha_{2}, \cdots, \alpha_{p-q}$ be the nonzero roots of $F^{\prime}(z)$ and let

$$
G(z)=\int_{0}^{z} \frac{z^{p-q} F^{\prime}(z) d z}{\prod_{i=1}^{p-q}\left(z-\alpha_{i}\right)\left(1-\bar{\alpha}_{i} z\right)}=d_{p} z^{p}+\cdots
$$

$G(z)$ is regular for $|z| \leqq 1$ and

$$
z G^{\prime}(z)=\frac{z^{p-q} z F^{\prime}(z)}{\prod_{i=1}^{p-q}\left(z-\alpha_{i}\right)\left(1-\bar{\alpha}_{i} z\right)}
$$

Since

$$
\arg \left[\frac{z^{p-q}}{\prod_{i=1}^{p-q}\left(z-\alpha_{i}\right)\left(1-\bar{\alpha}_{i} z\right)}\right]=0 \quad \text { for }|z|=1,
$$

$\arg z G^{\prime}(z)=\arg z F^{\prime}(z)$ for $|z|=1$.

Thus, $G(z)$ satisfies (2.9) and (2.10) on $|z|=1$. Hence, by Lemma 2, $G(z)$ is in $\mathscr{K}(p)$ and there exists $f(z)$ in $S(p)$, regular for $|z| \leqq 1$, such that 


$$
\operatorname{Re}\left[\frac{z G^{\prime}(z)}{f(z)}\right]>0 \quad(|z| \leqq 1) .
$$

But using the same reasoning as above, we have

$$
\arg \left[\frac{z G^{\prime}(z)}{f(z)}\right]=\arg \left[\frac{z F^{\prime}(z)}{f(z)}\right] \text { on }|z|=1 .
$$

Hence,

$$
\operatorname{Re}\left[\frac{z F^{\prime}(z)}{f(z)}\right]>0 \text { for }|z|=1 .
$$

Thus, $F(z)$ is in $\mathscr{K}(p)$.

Theorem 3 immediately gives us the following lemma, which will prove useful in obtaining a bound for the $(p+1)$ st coefficient of a function in $\mathscr{K}(p)$.

LEMma 3. If $F(z)$ is regular in $|z| \leqq 1$ and in $\mathscr{K}(p)$, then there exists

$$
f(z)=b_{p} z^{p}+\cdots \quad\left(\left|b_{p}\right|=1\right)
$$

regular and in $S(p)$ for $|z| \leqq 1$, such that

$$
\operatorname{Re}\left[\frac{z F^{\prime}(z)}{f(z)}\right]>0 \quad \text { on }|z|=1 .
$$

3. Some extremal problems for the class $\mathscr{K}(p)$. The following lemma has been proven by Royster [11]. However, the proof we give, which was communicated to me by Professor M. S. Robertson, seems to be different.

Lemma 4. Let $f(z)=[h(z)]^{-p}$, where $h(z)$ is in $S(1), h(0)=0, h^{\prime}(0)=1$ and let

$$
f(z)=\sum_{n=-p}^{\infty} C_{n} z^{n} \quad\left(0<|z|<1, C_{-p}=1\right),
$$

then

$$
\left|C_{n}\right| \leqq\left(\begin{array}{c}
2 p \\
n+p
\end{array}\right) \quad(n=-p, \cdots, 1),
$$

and these inequalities are sharp.

Proof. We write

$$
z^{p} f(z)=z^{p}[h(z)]^{-p}=\sum_{n=0}^{\infty} d_{n} z^{n} \quad\left(|z|<1, d_{0}=1\right) .
$$

The lemma will then be proven, if we can show 


$$
\left|d_{n}\right| \leqq\left(\begin{array}{c}
2 p \\
n
\end{array}\right) \quad(n \leqq p+1) .
$$

Taking the logarithm of both sides of (3.1), differentiating and multiplying through by $z$, we obtain

$$
-\frac{z f^{\prime}(z)}{p f(z)}=\frac{z h^{\prime}(z)}{h(z)} .
$$

Thus, we have for $|z|<1$

$$
\operatorname{Re}\left[-\frac{z f^{\prime}(z)}{p f(z)}\right]=\operatorname{Re}\left[\frac{z h^{\prime}(z)}{h(z)}\right]>0 \quad(|z|<1) .
$$

Let

$$
P(z)=-\frac{z f^{\prime}(z)}{p f(z)},
$$

then

$$
\operatorname{Re}\left[\frac{1}{P(z)}\right]>0 \quad \text { for }|z|<1
$$

Let

$$
\begin{aligned}
\frac{1}{P(z)} & =1+\sum_{n=1}^{\infty} \mu_{n} z^{n}, \\
\frac{1}{P(z)} & =-\frac{p f(z)}{z f^{\prime}(z)}=-\frac{p z^{p} f(z)}{z^{p+1} f^{\prime}(z)}, \\
-\frac{1}{P(z)} z^{p+1} f^{\prime}(z) & =p z^{p} f(z),
\end{aligned}
$$

or

$$
\left[-\sum_{m=0}^{\infty} \mu_{m} z^{m}\right]\left[\sum_{s=0}^{\infty}(s-p) d_{s} z^{s}\right]=p \sum_{n=0}^{\infty} d_{n} z^{n}
$$

Equating coefficients, we obtain

$$
\begin{aligned}
& p d_{n}=\sum_{r=0}^{n}(p-r) d_{r} \mu_{n-r}, \\
& n d_{n}=\sum_{r=0}^{n-1}(p-r) d_{r} \mu_{n-r} .
\end{aligned}
$$

Since $\left|\mu_{n-r}\right| \leqq 2$, we obtain

$$
n\left|d_{n}\right| \leqq 2 \sum_{r=0}^{n-1}(p-r)\left|d_{r}\right|
$$


provided $p-r \geqq 0$. That is, provided $n \leqq p+1$. Using (3.3) and a simple induction argument, we have

$$
\left|d_{n}\right| \leqq\left(\begin{array}{c}
2 p \\
n
\end{array}\right) \text { for } n \leqq p+1 .
$$

That the inequalities are sharp is shown by the function

$$
f(z)=\left[\frac{z}{(1+z)^{2}}\right]^{-p} .
$$

THEOREM 4. Let

$$
F(z)=\sum_{n=1}^{\infty} a_{n} z^{n} \quad(|z|<1)
$$

be regular and in $\mathscr{K}(p)$ for $|z|<1$, then

$$
\left|a_{p+1}\right| \leqq \sum_{k=1}^{p} \frac{2 k(2 p+1) !}{(p+k) !(p-k) !\left[(p+1)^{2}-k^{2}\right]}\left|a_{k}\right|
$$

and this inequality is sharp in all the variables $\left|a_{1}\right|, \cdots,\left|a_{p}\right|$.

REMARK. This theorem was first proven for $p=1$ be Reade [8].

Proof. We may assume without loss of generality that $F(z)$ is regular for $|z| \leqq 1$. Then, by Lemma 3 there exists a function

$$
f(z)=b_{p} z^{p}+\cdots \quad\left(\left|b_{p}\right|=1\right),
$$

regular for $|z| \leqq 1$ and in $S(p)$, such that

$$
\operatorname{Re}\left[\frac{z F^{\prime}(z)}{f(z)}\right]>0 \quad(|z|=1) .
$$

We may assume that $b_{p}=1$ since $\arg \left[b_{p}\right]$ is not involved in the inequality to be obtained. Thus we may write $f(z)$ in the form $[\phi(z)]^{p}$, where

$$
\phi(z)=z+\sum_{n=2}^{\infty} h_{n} z^{n}
$$

is regular for $|z|<1$ and in $S(1)$.

We may then write (3.5) in the form

$$
\operatorname{Re}\left[z F^{\prime}(z)[\phi(z)]^{-p}\right]>0 \quad \text { on }|z|=1 .
$$

Let

$$
[\phi(z)]^{-p}=\sum_{n=-p}^{\infty} C_{n} z^{n} \quad\left(0<|z|<1, C_{-p}=1\right) .
$$

Then 


$$
\begin{aligned}
z F^{\prime}(z)[\phi(z)]^{-p} & =\left[\sum_{n=1}^{\infty} n a_{n} z^{n}\right]\left[\sum_{n=-p}^{\infty} C_{n} z^{n}\right] \\
& =\sum_{k=-(p-1)}^{\infty} d_{k} z^{k},
\end{aligned}
$$

where

$$
d_{k}=\sum_{n=1}^{p+k} C_{-(n-k)} n a_{n} \quad(k=-(p-1), \cdots) .
$$

Consider the function $G(z)$ given by

$$
\begin{aligned}
G(z)=z F^{\prime}(z)[\phi(z)]^{-p} & -\sum_{k=-(p-1)}^{-1} d_{k} z^{k} \\
& +\sum_{k=-(p-1)}^{-1} \bar{d}_{k} z^{-k}
\end{aligned}
$$

Since $\bar{z}=z^{-1}$ for $|z|=1$, the last two terms in (3.6) add up to a purely imaginary number for $|z|=1$. Thus,

$$
\operatorname{Re}[G(z)]=\operatorname{Re}\left[z F^{\prime}(z)[\phi(z)]^{-p}\right]>0 \text { for }|z|=1 .
$$

But $G(z)$ is regular for $|z| \leqq 1$. Therefore,

$$
\operatorname{Re}[G(z)]>0 \text { for }|z| \leqq 1 \text {. }
$$

Now

$$
G(z)=d_{0}+\left(d_{1}+\bar{d}_{-1}\right) z+\cdots \quad(|z| \leqq 1) .
$$

Hence

$$
\begin{gathered}
\left|d_{1}+\bar{d}_{-1}\right| \leqq 2 \operatorname{Re}\left[d_{0}\right] \leqq 2\left|d_{0}\right| \\
\left|\sum_{n=1}^{p+1} C_{-(n-1)} n a_{n}+\sum_{n=1}^{p-1} \bar{C}_{-(n+1)} n \bar{a}_{n}\right| \leqq 2\left|\sum_{n=1}^{p} C_{-n} n a_{n}\right| \\
(p+1)\left|a_{p+1}\right| \leqq \sum_{n=1}^{p-1}\left[2 n\left|C_{-n}\right|+n\left|C_{-(n-1)}\right|+n\left|C_{-(n+1)}\right|\right]\left|a_{n}\right| \\
+\left[2 p\left|C_{-p}\right|+p\left|C_{-(p-1)}\right|\right]\left|a_{p}\right|
\end{gathered}
$$

By Lemma 4

$$
\left|C_{-k}\right| \leqq\left(\begin{array}{c}
2 p \\
p-k
\end{array}\right) \quad(k=1,2, \cdots, p)
$$

Therefore, 


$$
\begin{aligned}
(p+1)\left|a_{p+1}\right| \leqq \sum_{n=1}^{p-1}\left[2 n\left(\begin{array}{c}
2 p \\
p-n
\end{array}\right)\right. & \left.+n\left(\begin{array}{c}
2 p \\
p-n+1
\end{array}\right)+n\left(\begin{array}{c}
2 p \\
p-n-1
\end{array}\right)\right]\left|a_{n}\right| \\
& +\left[2 p+p\left(\begin{array}{c}
2 p \\
1
\end{array}\right)\right]\left|a_{p}\right| \\
& =(p+1) \sum_{n=1}^{p} \frac{2 n(2 p+1) !}{(p+n) !(p-n) !\left[(p+1)^{2}-n^{2}\right]}\left|a_{n}\right|
\end{aligned}
$$

which is (3.4).

We remark that the inequality is sharp, since it is known to be sharp for $f(z)$ in $S(p)$ with real coefficients [2], [4].

In order to obtain bounds on $\left|F^{\prime}(z)\right|$ for $F(z)$ in $\mathscr{K}(p)$, we will make use of the following lemma.

LEMMA 5. Let

$$
F(z)=a_{q} z^{q}+\cdots \quad(|z| \leqq 1)
$$

be regular and in $\mathscr{K}(p)$ for $|z| \leqq 1$. Let $\alpha_{1}, \alpha_{2}, \cdots, \alpha_{p-q}$ be the nonzero critical points of $F^{\prime}(z)$ in $|z|<1$. Then the function

$$
H(z)=\int_{0}^{z} z^{p-q} F^{\prime}(z)\left[\prod_{i=1}^{p-q}\left(\frac{\alpha_{i}}{\left|\alpha_{i}\right|}-\frac{z}{\left|\alpha_{i}\right|}\right)\left(\overline{\alpha_{i}} z-1\right)\right]^{-1} d z
$$

is regular for $|z| \leqq 1$ and in $\mathscr{K}(p)$.

Proof. By Lemma 3, there exists

$$
h(z)=b_{p} z^{p}+\cdots \quad\left(\left|b_{p}\right|=1\right),
$$

regular and in $S(p)$ for $|z| \leqq 1$, such that

$$
\begin{aligned}
\operatorname{Re} \frac{z F^{\prime}(z)}{h(z)}>0 \text { for }|z|=1 \\
\frac{z H^{\prime}(z)}{h(z)}=\frac{z^{p-q} z F^{\prime}(z)\left[\prod_{i=1}^{p-q}\left(\frac{\alpha_{i}}{\left|\alpha_{i}\right|}-\frac{z}{\left|\alpha_{i}\right|}\right)\left(\bar{\alpha}_{i} z-1\right)\right]^{-1}}{h(z)} .
\end{aligned}
$$

But,

$$
\arg \left(z^{p-q}\left[\prod_{i=1}^{p-q}\left(\frac{\alpha_{i}}{\left|\alpha_{i}\right|}-\frac{z}{\left|\alpha_{i}\right|}\right)\left(\bar{\alpha}_{i} z-1\right)\right]^{-1}\right)=0 \quad \text { on }|z|=1 .
$$

Thus,

$$
\frac{z H^{\prime}(z)}{h(z)}=M \frac{z F^{\prime}(z)}{h(z)}, \quad M>0 \quad \text { on }|z|=1
$$

Hence, 


$$
\operatorname{Re}\left[\frac{z H^{\prime}(z)}{h(z)}\right]>0 \text { for }|z|=1 .
$$

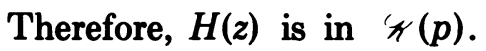

Theorem 5. Let

$$
F(z)=a_{q} z^{q}+\cdots \quad(|z|<1),
$$

be regular and in $\sharp(p)$ for $|z|<1$. Let $\alpha_{1}, \alpha_{2}, \cdots, \alpha_{p-q}$ be the nonzero critical points of $F(z)$ and let $\rho=\max \left|\alpha_{i}\right|$ and $\rho^{*}=\min \left|\alpha_{i}\right|$. Then

$$
\left|F^{\prime}\left(r e^{i \psi}\right)\right| \leqq \frac{(1+r) r^{q-1}}{(1-r)^{2 p+1}} q\left|a_{q}\right|\left[\prod_{i=1}^{p-q}\left(1+\frac{r}{\left|\alpha_{i}\right|}\right)\left(1+r\left|\alpha_{i}\right|\right)\right]
$$

$$
\left|F^{\prime}\left(r e^{i v}\right)\right| \geqq \frac{(1-r) r^{q-1}}{(1+r)^{2 p+1}} q\left|a_{q}\right|\left[\prod_{i=1}^{p-q}\left(\frac{r}{\left|\alpha_{i}\right|}-1\right)\left(1-r\left|\alpha_{i}\right|\right)\right]
$$$$
(\rho<r<1) \text {, }
$$

$$
\left|F^{\prime}\left(r e^{i v}\right)\right| \geqq \frac{(1-r) r^{q-1}}{(1+r)^{2 p+1}} q\left|a_{q}\right|\left[\prod_{i=1}^{p-q}\left(1-\frac{r}{\left|\alpha_{i}\right|}\right)\left(1-r\left|\alpha_{i}\right|\right)\right]
$$

$\left(r<\rho^{*}\right)$.

All these inequalities are sharp, equality being attained by the function

$$
F_{0}(z)=\int_{0}^{z} \frac{(1+z) z^{q-1}}{(1-z)^{2 p+1}} q\left|a_{q}\right| \prod_{i=1}^{p-q}\left(1+\frac{z}{\left|\alpha_{i}\right|}\right)\left(1+z\left|\alpha_{i}\right|\right) d z .
$$

Note that inequality (3.7) was obtained by Umezawa [13] for his class of $p$-valent close-to-convex functions.

Proof. We may assume without loss of generality that $F(z)$ is regular for $|z| \leqq 1$. Consider the functions $H(z)$ and $h(z)$, given in Lemma 5 and in its proof.

$$
\frac{z H^{\prime}(z)}{h(z)}=d_{0}+d_{1} z+\cdots \quad(|z| \leqq 1),
$$

where

$$
d_{0}=\frac{q a_{q}}{b_{p}}\left[\prod_{i=1}^{p-q}\left(-e^{i \arg \alpha_{i}}\right)\right]^{-1} .
$$

Then

$$
\frac{1}{\operatorname{Re}\left[d_{0}\right]}\left[\frac{z H^{\prime}(z)}{h(z)}-i \operatorname{Im}\left[d_{0}\right]\right]=P(z),
$$

where $\operatorname{Re} P(z)>0$ for $|z|<1$ and $P(0)=1$. Thus, 


$$
\left|\frac{P(z)-1}{P(z)+1}\right| \leqq|z|
$$

Hence

$$
\begin{aligned}
& \left|\frac{\frac{z H^{\prime}(z)}{h(z)}-d_{0}}{\frac{z H^{\prime}(z)}{h(z)}+\bar{d}_{0}}\right| \leqq|z|=r, \\
& (1-r)\left|\frac{z H^{\prime}(z)}{h(z)}\right| \leqq(1+r)\left|d_{0}\right|=(1+r) q\left|a_{q}\right| .
\end{aligned}
$$

Using the known bound

$$
|h(z)| \leqq \frac{r^{p}}{(1-r)^{2 p}} \text { for }|z|=r
$$

and using the definition of $H(z)$, we obtain

$$
\begin{aligned}
\left|F^{\prime}\left(r e^{i v}\right)\right| & \leqq \frac{(1+r)}{(1-r) r^{p-q+1}} q\left|a_{q}\right||h(z)|\left|\prod_{i=1}^{p-q}\left(\frac{\alpha_{i}}{\left|\alpha_{i}\right|}-\frac{z}{\left|\alpha_{i}\right|}\right)\left(\overline{\alpha_{i}} z-1\right)\right| \\
& \leqq \frac{(1+r) r^{q-1}}{(1-r)^{2 p+1}} q\left|a_{q}\right| \prod_{i=1}^{p-q}\left(1+\frac{r}{\left|\alpha_{i}\right|}\right)\left(1+r\left|\alpha_{i}\right|\right),
\end{aligned}
$$

which is (3.7).

To obtain (3.8) and (3.9), we notice that for $z=r e^{i \theta}$

$$
\begin{aligned}
&\left|\frac{P(z)+1}{P(z)-1}\right| \geqq \frac{1}{r}, \\
&|h(z)| \geqq \frac{r^{p}}{(1+r)^{2 p}}, \\
&\left|\frac{\alpha_{i}}{\left|\alpha_{i}\right|}-\frac{z}{\left|\alpha_{i}\right|}\right|\left|\overline{\alpha_{i}} z-1\right| \geqq\left(\frac{r}{\left|\alpha_{i}\right|}-1\right)\left(1-r\left|\alpha_{i}\right|\right) \quad\left(\left|\alpha_{i}\right|<r\right),
\end{aligned}
$$

and

$$
\left|\frac{\alpha_{i}}{\left|\alpha_{i}\right|}-\frac{z}{\left|\alpha_{i}\right|}\right|\left|\bar{\alpha}_{i} z-1\right| \geqq\left(1-\frac{r}{\left|\alpha_{i}\right|}\right)\left(1-r\left|\alpha_{i}\right|\right) \quad\left(r<\left|\alpha_{i}\right|\right) .
$$

Going through the same type of argument as before, we obtain (3.8) and (3.9).

The function $F_{0}(z)$ is in $\mathscr{K}(p)$ relative to

$$
f(z)=\frac{z^{q}}{(1-z)^{2}} \prod_{i=1}^{p-q}\left(1+\frac{z}{\left|\alpha_{i}\right|}\right)\left(1+z\left|\alpha_{i}\right|\right) .
$$


Equality in (3.7) is attained by $F_{0}^{\prime}(r)$, in (3.8) by $F_{0}^{\prime}(-r), r>\rho$, and in (3.9) by $F_{0}^{\prime}(-r), r<\rho^{*}$.

4. Radii of close-to-convexity and convexity for functions in $\mathscr{K}(p)$. Goodman [4] has proven that if

$$
f(z)=a_{q} z^{q}+\cdots \quad(|z|<1)
$$

is in $S(p)$, then

$$
\operatorname{Re} \frac{z f^{\prime}(z)}{f(z)} \geqq J_{q}(r) \text { for } r<\rho,
$$

where

$$
J_{q}(r)=q-r\left[\frac{2 p}{1+r}+\sum_{i=1}^{p-q} \frac{1}{\left|\alpha_{i}\right|-r}+\frac{\left|\alpha_{i}\right|}{1-\left|\alpha_{i}\right| r}\right],
$$

$\alpha_{1}, \cdots, \alpha_{p-q}$ being the nonzero roots of $f(z)$ and $\rho=\min \left|\alpha_{i}\right| . J_{q}(r)$ is a decreasing function of $r$ for $r<\rho$, is positive for $r=0$ and tends to $-\infty$ as $r$ tends to $\rho$. Thus, $J_{q}(r)$ has a least positive root $r_{q}$ and $J_{q}(r)>0$ for $r<r_{q}$.

We thus have that $f(z)$ is $q$-valently star-like for $|z|<r_{q}$. This estimate is sharp, since (4.1) was shown to be sharp [4], equality being attained at $z=-r$ by the function

$$
f(z)=z^{q}(1-z)^{-2 p} \prod_{i=1}^{p-q}\left(1+\frac{z}{\left|\alpha_{i}\right|}\right)\left(1+z\left|\alpha_{i}\right|\right) .
$$

THEOREM 6. Let

$$
F(z)=a_{q} z^{q}+\cdots \quad(|z|<1)
$$

be in $\mathscr{K}(p)$. Let $\alpha_{1}, \cdots, \alpha_{p-q}$ be the nonzero roots of $F^{\prime}(z)$ and let $r_{q}$ be the least positive root of $J_{q}(r)$, defined in (4.1). Then $F(z)$ is q-valently close-to-convex for $|z|<r_{q}$.

Proof. We first prove the theorem for $F(z)$, regular on $|z|=1$. Then there exists

$$
f(z)=b_{p} z^{p}+\cdots \quad(|z| \leqq 1),
$$

regular and in $S(p)$ for $|z| \leqq 1$, such that

$$
\operatorname{Re}\left[\frac{z F^{\prime}(z)}{f(z)}\right]>0 \quad \text { on }|z|=1 \text {. }
$$

Since

$$
\arg \left(z^{p-q}\left[\prod_{i=1}^{p-q}\left(z-\alpha_{i}\right)\left(1-\bar{\alpha}_{i} z\right)\right]^{-1}\right)=0 \quad \text { on }|z|=1,
$$

we have 
1965]

$$
\operatorname{Re}\left[\frac{z^{p-q} z F^{\prime}(z)}{\prod_{i=1}^{p-q}\left(z-\alpha_{i}\right)\left(1-\bar{\alpha}_{i} z\right) \cdot f(z)}\right]>0 \text { for }|z| \leqq 1 .
$$

Let

$$
g(z)=z^{q-p}\left[\prod_{i=1}^{p-q}\left(z-\alpha_{i}\right)\left(1-\bar{\alpha}_{i} z\right)\right] f(z) .
$$

Then, $g(z)$ is in $S(p)$ since $\operatorname{Re}\left[z g^{\prime}(z) / g(z)\right]>0$ on $|z|=1$. But $g(z)$ has nonzero roots at $\alpha_{1}, \alpha_{2}, \cdots, \alpha_{p-q}$. Therefore, $g(z)$ is $q$-valently star-like for $|z|<r_{q}$. Since

$$
\operatorname{Re}\left[\frac{z F^{\prime}(z)}{g(z)}\right]>0 \quad \text { for }|z| \leqq r_{q},
$$

$F(z)$ is $q$-valently close-to-convex for $|z|<r_{q}$.

If $F(z)$ is not regular on $|z|=1$, there exists a $\rho^{*}<1$ such that for $\rho^{*}$ $<\delta<1$ the function $G_{\delta}(z)=F(\delta z)$ is in $\pi(p)$ and regular on $|z|=1$. $G_{\delta}^{\prime}(z)=0$ for $z=\alpha_{i} / \delta$. Thus, $G_{\delta}(z)$ is $q$-valently close-to-convex for $|z|$ $<r_{q, \delta}$, where $r_{q, \delta}$ is the least positive root of

$$
J_{q, \delta}(r)=q-r\left[\frac{2 p}{1+r}+\sum_{i=1}^{p-q} \frac{\delta}{\left|\alpha_{i}\right|-r \delta}+\frac{\left|\alpha_{i}\right|}{\delta-\left|\alpha_{i}\right| r}\right] \text {. }
$$

Thus, there exists

$$
f_{\delta}(z)=C_{q} z^{q}+\cdots \quad\left(|z|<r_{q, \delta},\left|C_{q}\right|=1\right)
$$

$q$-valently star-like for $|z|<r_{q, \delta}$, such that

$$
\operatorname{Re}\left[\frac{z G_{\delta}^{\prime}(z)}{f_{\delta}(z)}\right]>0 \text { for }|z|<r_{q, \delta} .
$$

But $r_{q, \delta} \geqq r_{q}$, since $J_{q, \delta}(r) \geqq J_{q}(r)$ for $r<\min \left|\alpha_{i}\right|$. Thus $f_{\delta}(z)$ is $q$-valently star-like for $|z|<r_{q}$.

By a result of $M$. Cartwright [1] the family of $q$-valent functions $f(z)$ $=a_{q} z^{q}+\cdots\left(\left|a_{q}\right|=1\right)$ is a normal family. Thus we may choose an increasing sequence $\delta_{i}$ tending to 1 , such that the functions $f_{\delta_{i}}(z)$ tend to a function $f(z)$, which is $q$-valently star-like for $|z|<r_{q}$. Since for each $i$

$$
\operatorname{Re}\left[\frac{z G_{\delta_{i}}^{\prime}(z)}{f_{\delta_{i}}(z)}\right]>0 \text { for }|z|<r_{q}
$$

and since $z G_{\delta_{i}}^{\prime}(z)$ tends to $z F^{\prime}(z)$, we have

$$
\operatorname{Re}\left[\frac{z F^{\prime}(z)}{f(z)}\right] \geqq 0 \quad \text { for }|z|<r_{q} .
$$


Thus either $\operatorname{Re}\left[z F^{\prime}(z) / f(z)\right]>0$ for $|z|<r_{q}$, in which case $F(z)$ is $q$-valently close-to-convex for $|z|<r_{q}$, or $\left[z F^{\prime}(z) / f(z)\right]$ reduces to a constant for $|z|$ $<r_{q}$. In the second case $F(z)$ is $q$-valently convex and hence $q$-valently close-to-convex for $|z|<r_{q}$.

THEOREM 7. Let

$$
F(z)=a_{q} z^{q}+\cdots \quad(|z|<1),
$$

be in $\mathscr{K}(p)$, then $F(z)$ is q-valently convex for $|z|<\beta_{q}$, where $\beta_{q}$ is the least positive root of

$$
K_{q}(r)=J_{q}(r)-\frac{2 r}{1-r^{2}}
$$

and this estimate is the best possible.

Proof. Let us first assume that $F(z)$ is regular on $|z|=1$. Then, as we have seen before, there exists

$$
g(z)=b_{q} z^{q}+\cdots \quad(|z|<1),
$$

which is in $S(p)$ for $|z|<1$, such that

$$
\operatorname{Re}\left[\frac{z F^{\prime}(z)}{g(z)}\right]>0 \text { for }|z| \leqq 1 .
$$

Let

$$
\begin{aligned}
\frac{z F^{\prime}(z)}{g(z)} & =P(z), \quad \operatorname{Re}[P(z)]>0 \quad \text { for }|z| \leqq 1, \\
1+\frac{z F^{\prime \prime}(z)}{F^{\prime}(z)} & =\frac{z P^{\prime}(z)}{P(z)}+\frac{z g^{\prime}(z)}{g(z)} .
\end{aligned}
$$

Now $g(z)$ has the same zeros as $F^{\prime}(z)$. Therefore,

$$
\operatorname{Re}\left[\frac{z g^{\prime}(z)}{g(z)}\right] \geqq J_{q}(r) \quad \text { for } r<\min \left|\alpha_{i}\right| .
$$

By a result, obtained independently by Libera [6], MacGregor [7] and Robertson [10], we have

$$
\operatorname{Re}\left[\frac{z P^{\prime}(z)}{P(z)}\right] \geqq-\frac{2 r}{1-r^{2}} .
$$

Thus

$$
\operatorname{Re}\left[1+\frac{z F^{\prime \prime}(z)}{F^{\prime}(z)}\right] \geqq-\frac{2 r}{1-r^{2}}+J_{q}(r)=K_{q}(r)
$$

for $r<\min \left|\alpha_{i}\right|$.

Thus, if $|z|<\beta_{q}$ 


$$
\operatorname{Re}\left[1+\frac{z F^{\prime \prime}(z)}{F^{\prime}(z)}\right]>0
$$

Since $F^{\prime}(z)$ has $(q-1)$ zeros in $|z|<\beta_{q}$, all of them at the origin,

$$
\int_{0}^{2 \pi} \operatorname{Re}\left[1+\frac{z F^{\prime \prime}(z)}{F^{\prime}(z)}\right] d \theta=2 q \pi \quad\left(|z|<\beta_{q}\right) .
$$

Thus $F(z)$ is $q$-valently convex for $|z|<\beta_{q}$.

Arguing as in Theorem 6, we may remove the assumption of regularity on $|z|=1$.

The function

$$
F(z)=\int_{0}^{z} \frac{(1+z) z^{q-1}}{(1-z)^{2 p+1}} \prod_{i=1}^{p-q}\left(1+\frac{z}{\left|\alpha_{i}\right|}\right)\left(1+z\left|\alpha_{i}\right|\right) d z
$$

shows that the radius found is sharp, since

$$
1+\frac{z F^{\prime \prime}(z)}{F^{\prime}(z)}=K_{q}(r)
$$

for $z=-r, r<\min \left|\alpha_{i}\right|$.

\section{REFERENCES}

1. M. Cartwright, Some inequalities in the theory of functions, Math. Ann. 111 (1935), 98-118.

2. A. W. Goodman and M. S. Robertson, A class of multivalent functions, Trans. Amer. Math. Soc. 70 (1951), 127-136.

3. A. W. Goodman, On some determinants related to p-valent functions, Trans. Amer. Math. Soc. 63 (1948), 175-192.

4. , On the Schwarz-Christoffel transformation and p-valent functions, Trans. Amer. Math. Soc. 68 (1950), 204-223.

5. W. Kaplan, Close-to-convex schlicht functions, Michigan Math. J. 1 (1952), 169-185 (1953).

6. R. J. Libera, Radius of convexity problems, Thesis, Rutgers, The State University, New Brunswick, N. J., 1962.

7. T. H. MacGregor, Functions whose derivative has a positive real part, Trans. Amer. Math. Soc. 104 (1962), 532-537.

8. Maxwell O. Reade, The coefficients of close-to-convex functions, Duke Math. J. 23 (1956), 459-462.

9. M. S. Robertson, Multivalently star-like functions, Duke Math. J. 20 (1953), 539-550.

10. - Extremal problems for analytic functions with positive real part and applications, Trans. Amer. Math. Soc. 106 (1963), 236-253.

11. W. C. Royster, Meromorphic starlike multivalent functions, Trans. Amer. Math. Soc. 107 (1963), 300-308.

12. T. Umezawa, On the theory of univalent functions, Tôhoku Math. J. 7 (1955), 212-223.

13. , Multivalently close-to-convex functions, Proc. Amer. Math. Soc. 8 (1957), 869-874.

\section{LAfayette College,}

Easton, Pennsylvania 